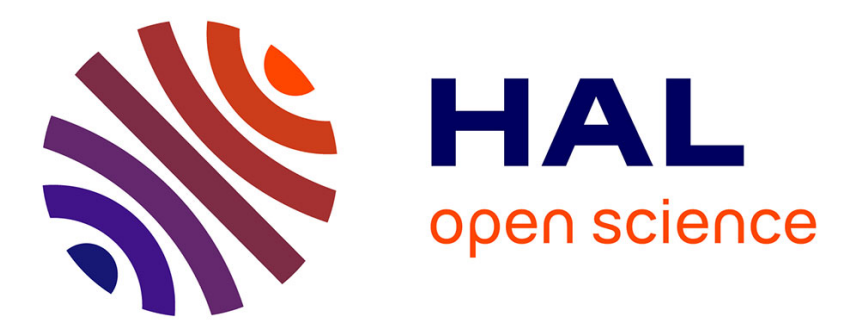

\title{
Design and Prototyping of a Partially Decoupled 4-DOF 3T1R Parallel Manipulator with High-Load Carrying Capacity
}

\author{
Sébastien Briot, Vigen Arakelian, Sylvain Guegan
}

\section{To cite this version:}

Sébastien Briot, Vigen Arakelian, Sylvain Guegan. Design and Prototyping of a Partially Decoupled 4-DOF 3T1R Parallel Manipulator with High-Load Carrying Capacity. Transaction of the ASME, Journal of Mechanical Design, 2008, 130 (12), pp.Vol. 130, No. 12. hal-00362518

\section{HAL Id: hal-00362518 https://hal.science/hal-00362518}

Submitted on 24 Jun 2019

HAL is a multi-disciplinary open access archive for the deposit and dissemination of scientific research documents, whether they are published or not. The documents may come from teaching and research institutions in France or abroad, or from public or private research centers.
L'archive ouverte pluridisciplinaire $\mathbf{H A L}$, est destinée au dépôt et à la diffusion de documents scientifiques de niveau recherche, publiés ou non, émanant des établissements d'enseignement et de recherche français ou étrangers, des laboratoires publics ou privés. 
Sébastien Briot

Département de Génie Mécanique et Automatique, L.G.C.G.M. EA3913

Institut National des Sciences Appliquées (I.N.S.A.) 20 avenue des buttes de Coësmes -

CS 14315

F-35043 Rennes, France sebastien.briot@ens.insa-rennes.fr

Vigen Arakelian

vigen.arakelyan@insa-rennes.fr

Sylvain Guégan

sylvain.guegan@insa-rennes.fr

\section{Design and Prototyping of a Partially Decoupled 4-DOF 3T1R Parallel Manipulator with High-Load Carrying Capacity}

In this paper, a new 4 degrees of freedom 3T1R parallel manipulator with high-load carrying capacity is presented. This manipulator generates Schönflies motions, in which the moving platform carries out three independent translations and one rotation about one axis of fixed orientation. The particularity of the proposed architecture is the decoupling of the displacements of the platform in the horizontal plane from its translation along the vertical axis. Such a decoupling allows the cancellation of the gravity loads on the actuators which displace the platform in the horizontal plane. A prototype of the proposed manipulator with four degrees of freedom and experimental validation of the suggested concept are also presented. Two cases have been examined on the built prototype: manipulator with payload and without. It was shown that the input torques of actuators displacing the platform in the horizontal plane for these two cases are the same, i.e. the payload does not bring any load to the actuators.

\section{Introduction}

The robots with varied architectures generate Schönflies motions (alternatively called SCARA motions or 3T1R motions), in which the end-effector carries out three independent translations and rotates around a fixed axis. The first robot of this type was developed at Japon's Yamanashi University [1]. This robot, named SCARA (Selective Compliance Assembly Robot Arm) is a high-performance system and nowadays, there are about 240 types of SCARA robots developed by 20 Companies. With the emergence of parallel manipulators, new structures have been proposed for carrying out Schönflies motions. One of the earliest parallel manipulators generating these motions was the Delta robot $[2,3]$. In this parallel architecture with only three-translation degrees of freedom, the rotation of the gripper is achieved by an additional actuator mounted on the frame. Later, the Kanuk and the Manta architectures were developed [4]. A contribution to the further development of the Delta robot was the manipulator H4 [5, 6], in which four legs are mounted on the frame making it possible to carry out 3T1R motions with 4 coupled actuators. At McGill University, several architectures based on parallelogram mechanisms were developed [7-9]. New architectures were proposed on the basis of systematic synthesis procedures [10-13]. However, both for the other parallel manipulators and for these architectures, a drawback is the high coupling of kinematics and dynamics. This non-linearity of the kinematic and dynamic models of parallel manipulators is not attractive for industrial applications. In order to solve this problem new structures have been developed over the last few years [14-19] in which the decoupling of the displacements in regard to all degrees of freedom of the platform is achieved.
Our observations have shown that despite rather-encouraging results, it is not easy to develop a parallel architecture with fullydecoupled motions and to conserve its principal advantages: the higher stiffness of the structure with light links. In the case of the fully decoupled parallel manipulators, the platform is connected to the base by several legs but each of them operates only in one direction, i.e. one leg carries the whole load. In other words, the parallel manipulator becomes a structure in which the payload is connected to several chains operating separately. There were also efforts to create partially decoupled parallel manipulators [20] but the noted drawbacks are the same.

Which is why we have tried to find a compromise between the decoupling of the movements and the architectural particularity of the parallel structures, i.e. the higher stiffness of the structure with light links. In other words, we have changed the statement of the problem: it is not essential that a parallel architecture will be fully-decoupled, it may also be partially decoupled but it is important to obtain a mechanical architecture with a good payload-to-weight ratio.

2 New concept of the partially decoupled parallel manipulator

An energetic analysis shows that the gravity work of a body moving in the horizontal plane is equal to zero (the gravitational forces are always perpendicular to the displacements). But the work of the same force moving along the vertical axis is other than zero (the gravitational forces are parallel to the displacements). This phenomenon is used in the design of the hand operated manipulators $[21,22]$, in which the horizontal 
displacements of the payload are carried out manually and the vertical displacements are actuated. This principle is applied in the design of the new parallel manipulators called PAMINSA ${ }^{1}$.

Let us consider the mechanical architecture of the suggested manipulators. The aim of this innovation was to develop a parallel architecture in which displacements of the platform in the horizontal plane are independent of its vertical displacements. For this purpose, the pantograph linkage is used as a leg. The pantograph is a mechanical system with two input points $A_{i}$ and $B_{i}$ and one output point $C_{i}$ (Fig. 1a). These input points linearly control the displacement of the output point $C_{i}$. Thus, one linear actuator connected with input point $B_{i}$ can control the vertical displacement of the output point $C_{i}$ and one other linear actuator with horizontal axis is able to control its horizontal displacements. Please note that these motions are completely decoupled, i.e. they can be carried out independently.

Now let us connect three Scheiner pantograph linkages with the base and the platform as shown in Fig. 1b. In the obtained structure, one vertical actuator $M_{v}$ controls the vertical displacement of point $B_{i}$ of the pantograph linkages, resulting in the vertical displacement of joints $C_{i}$ of the moving platform. The generation of motion in the horizontal plane is achieved by the actuators $M_{1}, M_{2}$ and $M_{3}$ moving the input joints $A_{i}$.

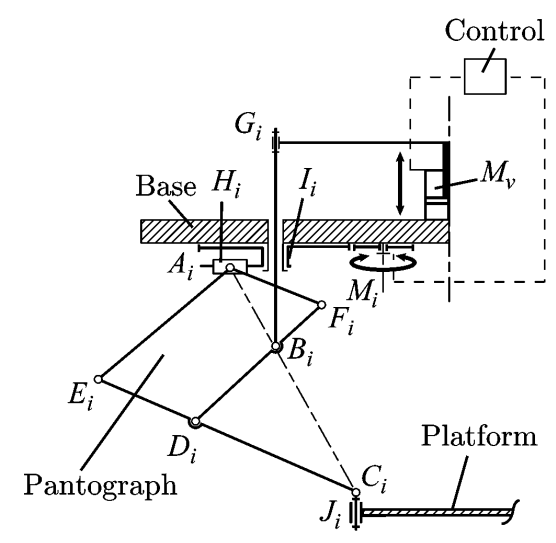

(a)

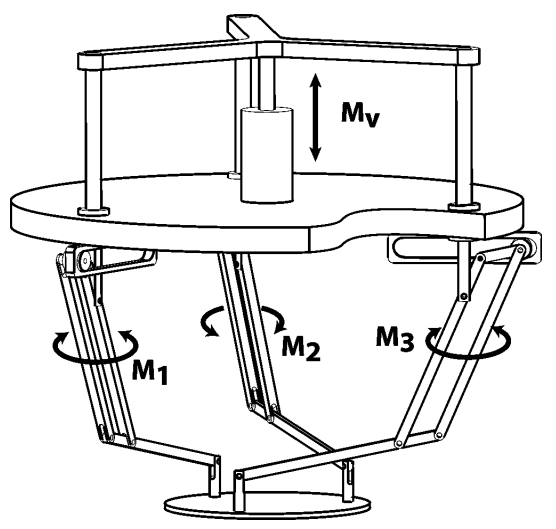

(b)

Figure 1. PAMINSA: a) kinematic chain of each leg; b) 3D view.

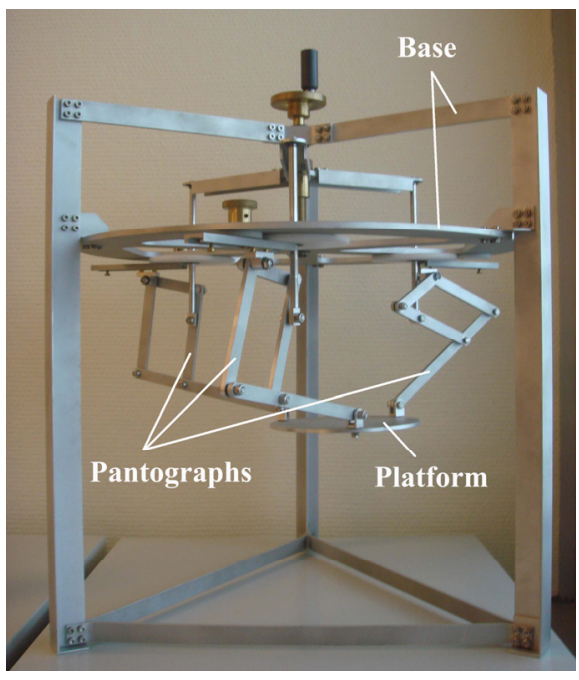

Figure 2. The first model of PAMINSA.

Fig. 2 shows the first hand-operated model of PAMINSA. It is easy to see that, in the suggested architecture, the vertical translation of the platform along $\boldsymbol{z}$ axis is decoupled from its displacements in the horizontal plane (translations along $\boldsymbol{x}$ and $\boldsymbol{y}$ axes and rotation $\phi$ around $z$ axis).

Among the obvious advantages of the suggested manipulator architecture, we would like to note the following:

(i) the decoupling of the control powers into two parts, making it possible to raise an important payload to a fixed altitude with powerful actuators and then to displace it on the horizontal plane with less powerful actuators;

(ii) a great accuracy in the horizontal positioning because the payload can be locked in the horizontal plane using mechanical architecture of the manipulator (in other words, if the position of the vertical actuator is fixed, the altitude of the platform cannot change);

the cancellation of the loads of gravity on the rotating actuators which displace the platform in the horizontal plane;

(iv) the simplification of the vertical control which is based on linear input/output relationships.

The next section will present the kinematics and statics of the suggested manipulator.

\section{Kinematic, singularity and static analysis}

\subsection{Kinematic and singularity analysis}

The kinematics of this manipulator is simple because its analysis can be decomposed into two parts. The displacement along the vertical axis is described by the well known copying properties of the pantograph: $z=k Z$, where $k$ is the magnification factor of the pantograph, $Z$ is the displacement of the input point $B_{i}$ and $z$ is the displacement of the output point $C_{i}$ of the pantograph (Fig. 1a). With regard to the displacements in the horizontal plane, the projection of the manipulator in the horizontal plane is similar to a $3-\underline{R} P R$ parallel manipulator (Fig. $3)$. In this case, the horizontal motions of the platform are produced by three actuators $M_{1}, M_{2}$ and $M_{3}$. The position and

${ }^{1}$ PAMINSA: $\underline{\text { Parallel }}$ Manipulator of the I.N.S.A. 
orientation of the mobile coordinate system of the platform in the fixed coordinate system $x O y$ can be represented by the vector $\mathbf{x}=(x, y, \phi)^{T}$, where $x, y$ are the coordinates of the center of the isosceles triangle $C_{1} C_{2} C_{3}, \phi$ is the angle of rotation around this center. The input parameters are expressed by the vector $\mathbf{q}=\left(\theta_{1}, \theta_{2}, \theta_{3}\right)^{T}$.

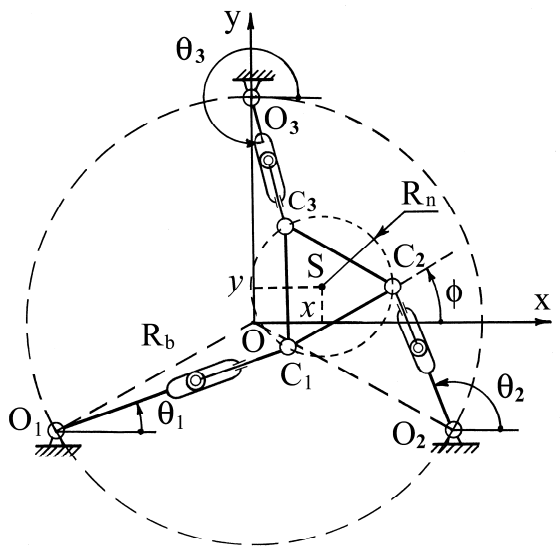

Figure 3. Projection of the manipulator in the horizontal plane.

The relations between the input $\mathbf{q}=\left(\theta_{1}, \theta_{2}, \theta_{3}\right)^{T}$ and output $\mathbf{x}=(x, y, \phi)^{T}$ parameters can be found from the closure loop equations:

$$
\begin{aligned}
& \cos \theta_{1}\left(-R_{p l} \cos \left(\phi+\frac{2 \pi}{3}\right)-R_{b} / 2-y\right) \\
& -\sin \theta_{1}\left(R_{p l} \sin \left(\phi+\frac{2 \pi}{3}\right)-\sqrt{3} R_{b} / 2-x\right)=0 \\
& \cos \theta_{2}\left(-R_{p l} \cos \left(\phi-\frac{2 \pi}{3}\right)-R_{b} / 2-y\right) \\
& -\sin \theta_{2}\left(-R_{p l} \sin \left(\phi-\frac{2 \pi}{3}\right)+\sqrt{3} R_{b} / 2-x\right)=0 \\
& \cos \theta_{3}\left(-R_{p l} \cos \phi+R_{b}-y\right)-\sin \theta_{3}\left(R_{p l} \sin \phi-x\right)=0
\end{aligned}
$$

where $R_{b}=l_{O O 1}=l_{O O 2}=l_{O O 3}$ and $R_{p l}=l_{S C 1}=l_{S C 2}=l_{S C 3}$.

The Jacobian matrix $\mathbf{J}$, expressing the relation between the vector of the time derivatives of the input joint coordinates and the times derivatives of the position of the platform, can be derived from the expressions (1-3) [23-26]:

$$
\mathbf{J}=\mathbf{A}^{-1} \mathbf{B}
$$

with

$\mathbf{A}=\left[\begin{array}{cccc}\rho_{1} & 0 & 0 & 0 \\ 0 & \rho_{2} & 0 & 0 \\ 0 & 0 & \rho_{3} & 0 \\ 0 & 0 & 0 & k\end{array}\right], \rho_{i}=O_{i} C_{i}$

$$
\mathbf{B}=\left[\begin{array}{cccc}
\sin \theta_{1} & -\cos \theta_{1} & R_{p l} \sin \left(\phi+2 \pi / 3-\theta_{1}\right) & 0 \\
\sin \theta_{2} & -\cos \theta_{2} & R_{p l} \sin \left(\phi-2 \pi / 3-\theta_{2}\right) & 0 \\
\sin \theta_{3} & -\cos \theta_{3} & R_{p l} \sin \left(\phi-\theta_{3}\right) & 0 \\
0 & 0 & 0 & 1
\end{array}\right]
$$

Thus, the singularity of parallel manipulators appears when these matrices are rank-deficient, i.e. when

$$
\begin{aligned}
\operatorname{det}(\mathbf{A})= & k \rho_{1} \rho_{2} \rho_{3}=0 \\
\operatorname{det}(\mathbf{B})=- & \left(R_{p l}-R_{b} \cos \phi\right) \\
& \left(x^{2}+y^{2}-\left(R_{p l}^{2}+R_{b}^{2}-2 R_{p l} R_{b} \cos \phi\right)\right) /\left(\rho_{1} \rho_{2} \rho_{3}\right)=0
\end{aligned}
$$

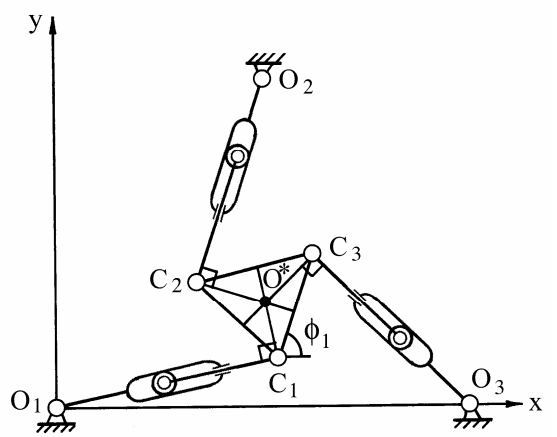

(a)

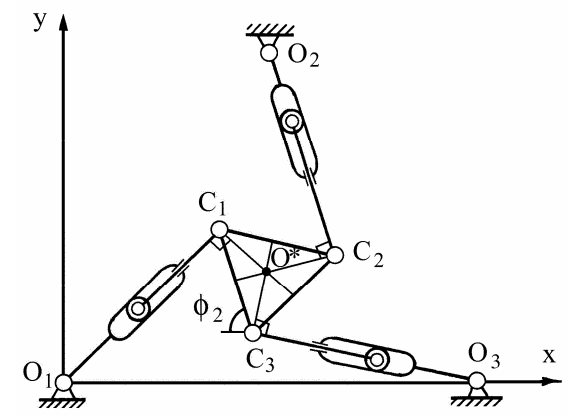

(b)

Figure 4 . Two singular positions corresponding to $\phi_{1,2}= \pm \cos ^{-1}\left(R_{p l} / R_{b}\right)$.

These conditions will be reached when:

a) $\rho_{1} \rho_{2} \rho_{3}=0$. These singular positions correspond to manipulator configurations in which the $C_{1}$ axis is aligned with the $O_{1}$ axis, the $C_{2}$ axis is aligned with the $O_{2}$ axis, and the $C_{3}$ axis is aligned with the $O_{3}$ axis, i.e. the axis $A_{\mathrm{i}}, B_{\mathrm{i}}$ and $C_{\mathrm{i}}$ are aligned along the vertical direction (see Fig. 1a). Please note that all the vertical axes passing through $C_{1}, C_{2}$ and $C_{3}$ axes are aligned simultaneously with the vertical axes passing through $O_{1}, O_{2}$ and $O_{3}$ axes when $R_{b}=R_{p l}$. In these configurations, unlimited values of parameters of $\theta_{1}, \theta_{2}$ and/or $\theta_{3}$ correspond to the given position of the platform, 
i.e. the motion of the input $\operatorname{link}(\mathrm{s})$ has no influence on the position of the platform.

b) $R_{p l}-R_{b} \cos \phi=0$. This condition is reached if $\phi_{1,2}= \pm \cos ^{-1}\left(R_{p l} / R_{b}\right)$. Two configurations corresponding to this singularity are shown in Fig. 4. In these positions, for fixed values of the active-joint variables $\theta_{1}, \theta_{2}$ and $\theta_{3}$, infinitesimal rotations around the center $O^{*}$ are possible.

c) $x^{2}+y^{2}-\left(R_{b}^{2}+R_{n}^{2}-2 R_{b} R_{n} \cos \phi\right)=0$. The manipulator configuration corresponding to this singularity is shown in Fig. 5. In this configuration, the center $S$ of the platform is on a circle of radius $\sqrt{R_{b}^{2}+R_{n}^{2}-2 R_{b} R_{n} \cos \phi}$ and for the fixed active-joint variables, the platform may generate infinitesimal rotation around center $O^{*}$.

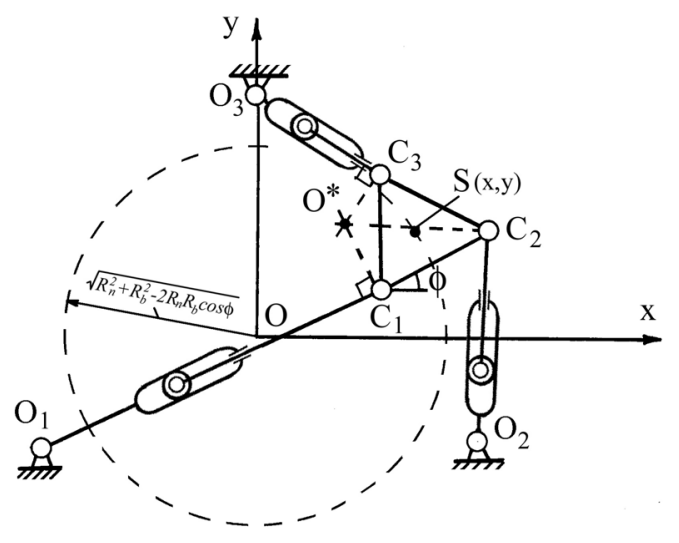

Figure 5. Singular configuration of the manipulator when the center $\mathbf{S}$ of the platform is on a circle of radius

$\sqrt{R_{b}^{2}+R_{n}^{2}-2 R_{b} R_{n} \cos \phi}$.

Other types of singularities, which are not described in the previous approach, are due to the degeneracy of the pantograph linkages. They can be found by analyzing the Jacobian matrix $\mathbf{J}_{(\mathbf{L E G}) i}$, defining the relations between the time derivatives of the coordinates of points $C_{i}$ (expressed in the plane of the pantograph) and the time derivatives of the angles $\varphi_{1 i}$ and $\varphi_{2 i}$, where, $\varphi_{1 i}$ is the angle between the directions of the prismatic pair $H_{i}$ and segment $B_{i} D_{i}$ and $\varphi_{2 i}$ is the angle between directions of segments $B_{i} D_{i}$ and $D_{i} C_{i}$ (Fig.1a):

$\mathbf{J}_{(\mathbf{L E G}) i}=\left[\begin{array}{cc}-l_{B_{i} D_{i}} \sin \varphi_{1 i}-l_{D_{i} C_{i}} \sin \left(\varphi_{1 i}+\varphi_{2 i}\right) & -l_{D_{i} C_{i}} \sin \left(\varphi_{1 i}+\varphi_{2 i}\right) \\ l_{B_{i} D_{i}} \cos \varphi_{1 i}+l_{D_{i} C_{i}} \cos \left(\varphi_{1 i}+\varphi_{2 i}\right) & l_{D_{i} C_{i}} \cos \left(\varphi_{1 i}+\varphi_{2 i}\right)\end{array}\right]$, $(i=1,2,3)$

Thus, the singularity appears when this matrix is rankdeficient, i.e. when

$\operatorname{det}\left(\mathbf{J}_{(\mathrm{LEG}) i}\right)=l_{B_{i} D_{i}} l_{D_{i} C_{i}} \sin \varphi_{2 i}=0$
These conditions will be reached when $l_{B_{i} D_{i} D_{i} C_{i}} \sin \varphi_{2 i}=0$, i.e. when link $B_{i} D_{i}$ is either fully stretched out or folded back on link $D_{i} C_{i}$

It should be noted that there are some particular cases of these singular configurations when finite translations or rotations can be produced (self-motions) [26].

\subsection{Static analysis}

With regard to the static analysis of the manipulator, the input torques/force $Q_{j}^{s t}$ applied to the actuators $M_{j}(j=1,2,3,4)$ due to the force of gravity of links, joints and platform of the studied manipulator can be expressed as:

$Q_{j}^{s t}=Q_{p l j}^{s t}+\sum_{p=1}^{3}\left(\sum_{i=1}^{n} Q_{i p j}^{s t}\right), \quad(j=1,2,3,4)$,

where $Q_{i p j}^{s t}$ is the load applied to the actuator $j$ due to the gravity of the $i$-th link or bearings of the $p$-th leg $(p=1,2,3)$, $Q_{p l j}^{s t}$ is the load applied to the actuator $j$ due to the gravity of the platform (see detailed expression in [27]).

These loads can be represented in the form:

$Q_{i p j}^{s t}(x, y, \phi, z)=\left(\sum_{p=1}^{3} \mathbf{J}_{i p}^{T}(x, y, \phi, z) \mathbf{G}_{i p}\right){ }_{j}$

$Q_{p l j}=\left.\left(\mathbf{J}^{T} \mathbf{G}_{p l}\right)\right|_{j}$

where $\mathbf{J}_{i p}$ is the Jacobian matrix between the point $P_{i p}$ and the actuated variables $\left[\theta_{1}, \theta_{2}, \theta_{3}, Z\right]$ (i.e. it relates the velocity of point $P_{i p}$ to the articular joints velocities), $P_{i p}$ is the center of masses of the $i$-th link, $\mathbf{J}$ is the general Jacobian matrix of the robot between the point $P$ and the actuated variables, $P$ is the center of masses of the platform, $\mathbf{G}$ and $\mathbf{G}_{i p}$ are respectively the forces of gravity of the platform and the links (or bearings).

It is easy to see that:

$Q_{p l j}=\left.\left(\mathbf{J}^{T} \mathbf{G}_{p l}\right)\right|_{j}=0$, for $j=1,2,3$.

i.e. the input torques of the rotating actuators due to the gravity of the platform are cancelled because the gravitational forces are always perpendicular to the displacements (the platform carry out the displacements in the horizontal plane).

It should be noted that as the studied architecture of the manipulator is symmetrical, the values of the input torques for the actuators are also symmetrical but they are situated in different zones (rotations of $\pm 120^{\circ}$ ).

Thus, the input torques of the rotating actuators taking into account the mass distribution of each leg can be written as following:

$Q_{j}^{s t}=\sum_{p=1}^{3}\left(\sum_{i=1}^{n} Q_{i p j}^{s t}(x, y, \phi, z)\right), \quad(j=1,2,3)$,

where 
$Q_{i p j}^{s t}(x, y, \phi, z)=\left(\sum_{p=1}^{3} \mathbf{J}_{i k}^{T}(x, y, \phi, z) \mathbf{G}_{i p}\right){ }_{j}$

It was also shown in [21] that the input torques due to the movable masses of the pantograph linkage may be cancelled by its optimal redistribution. Thus, by means of complete static balancing of legs, it is possible to cancel the loads due to the movable masses of the legs on the rotating actuators.

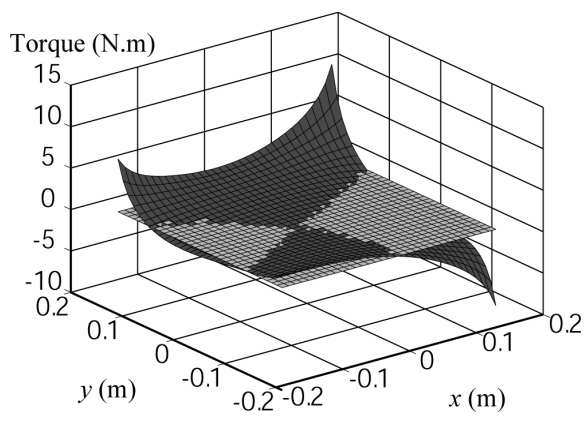

Fig. 6. Variations of the actuator torques for $\mathrm{z}=-\mathbf{0 . 6 \mathrm { m } \text { and } \phi =}$ $0^{\circ}$ before (dark grey) and after (bright grey) static balancing of legs.

Fig. 6 shows the variations of the actuator torques before and after mass balancing. After complete static balancing the potential energy of the manipulator is constant for any configuration and zero actuator torques are required. For example, the added masses are located at axes $F_{i}$ (Fig. 1a) and their values are $2.8 \mathrm{~kg}$.

\section{Prototype and experimental validation of the suggested design approach}

In order to validate the properties of a new parallel manipulator, we have developed a prototype (Fig. 7) at the I.N.S.A. in Rennes. The displacements on the horizontal plane of the developed prototype are obtained by Harmonic Drive motors connected with the legs by means of toothed-belt transmission.

As an actuator, which controls the linear displacement of the vertical axes of the manipulator's legs, a PARVEX motor system was chosen. The pantograph linkage has been carried out with double rods in order to increase the stiffness of the mechanical system. The pantograph links of the built prototype are hollow tubes with a thickness of $1.5 \mathrm{~mm}$. It is obvious that, for an industrial application, creating a pantograph with single and more rigid links is more attractive.

In order to validate the numerical simulations with experimental tests, we have measured the input torques/efforts of the actuators with and without a payload of $200 \mathrm{~N}$ (Fig. 7a, b) for the trajectory given in figure 8 . The static balancing of the manipulator is experimentally accomplished by adding counterweights of $2.8 \mathrm{~kg}$ at the axes $F_{i}$ to the pantograph linkages (Fig. 9).

The experimental bench could be seen on figure 10. It is composed of the PAMINSA robot with its control system, a computer to interact with the user and a dSPACE 1103 card. To control the system, the softwares Matlab/Simulink/RTI/ ControlDesk have been used. The sampling period was $1 \mathrm{~ms}$. For the motions in the horizontal plane, the PAMINSA is composed of

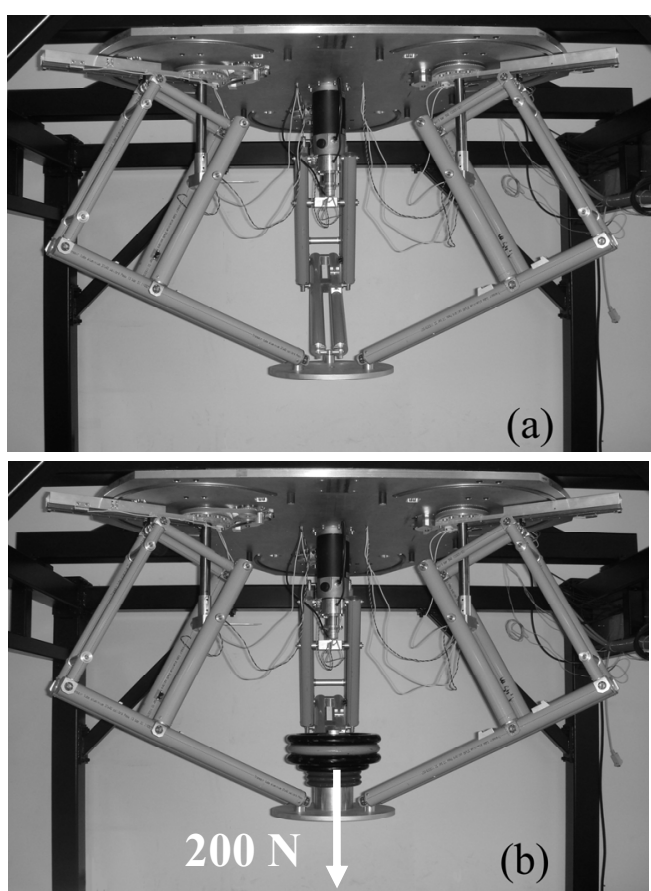

Figure 7. The prototype of PAMINSA developed in the I.N.S.A. of Rennes.
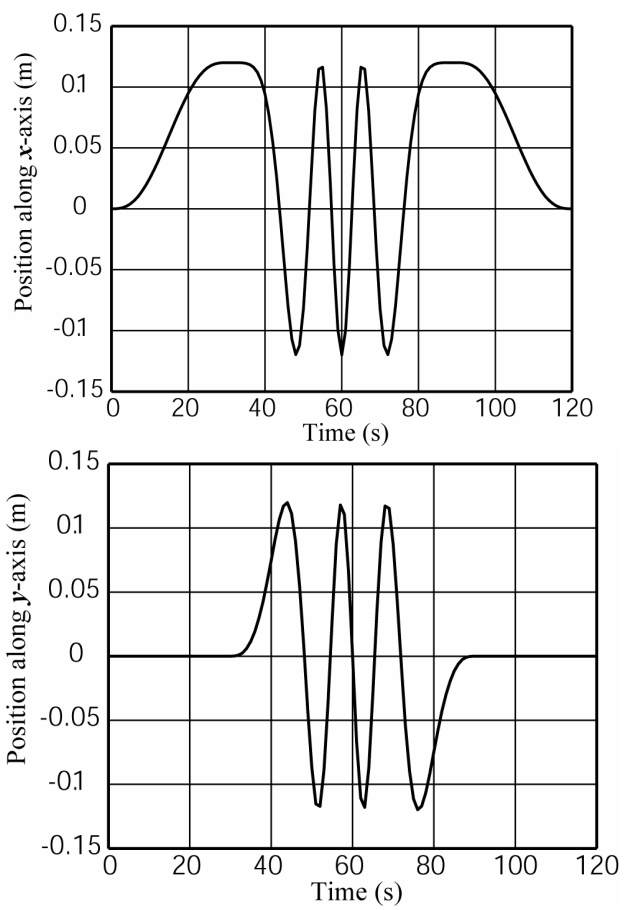

Figure 8. Position of the platform for $z=-0.6 \mathrm{~m}$ and $\phi=0^{\circ}$.

three identical Harmonic Drive DC motors (3557012CR) in combination with Fulhaber incremental encoders (HEDS5500) and Fulhaber motion controllers (MCDC2805). A Parvex DC motor, (RX320DR1200) including an encoder and a brake system is used for the translation along the vertical axis, in combination 
with an e-motion servo-amplifier (SCA-SS-70-10). The dSPACE 1103 carries out the interface between the $\mathrm{PC}$ and the servosystems. This card allows the control of the 4 motorized axes and has specific entries to which we have directly connected the 4 incremental encoders. We have also connected three LEM (HX10NP) current sensors to observe the torque of motor 1, 2 and 3.

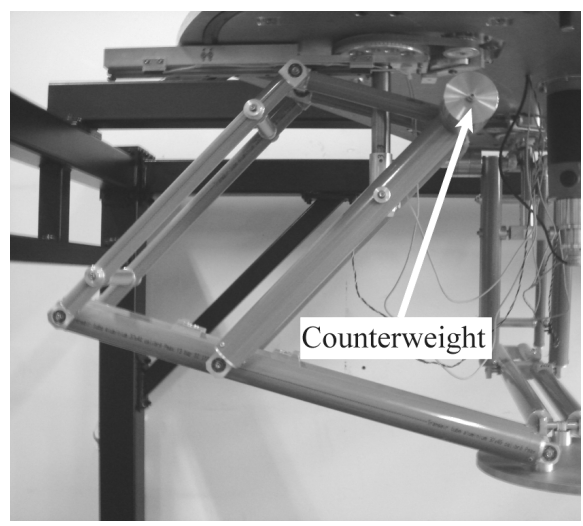

Figure 9. Counterweights mounted into the pantograph linkages.
The robot is controlled by the classical PI law directly implemented under Simulink/ControlDesk. In order to make it, each motion controller has been configured to be only used as power amplifiers. Thus, the motor voltage is proportional to the voltage at the analog input of each motion controller. Optics Sensors have been added for the initialization of the robot and for the setting up safety measures.

The obtained results are presented in figure 11. The numerical simulations were validated via these experimental tests. The curves with and without payload for the 3 rotating actuators (Fig. 11a, b, c) are superposed. We can see that they are similar, i.e. the loads on these actuators are cancelled. The small differences might result from friction in the joints, manufacturing errors, elasticity of the links or tracking errors.

Regarding the vertical actuator (Fig. 11d), it supports the payload and the increase of the input force is significant.

Thus, it can be noted that the obtained measures validate the proposed design approach

\section{Conclusion}

In this paper, we have presented a novel motion decoupling technique for parallel mechanisms and its application to the design of a new partially decoupled parallel manipulator called PAMINSA. The architecture of this parallel manipulator is composed of three legs, which are pantograph linkages.

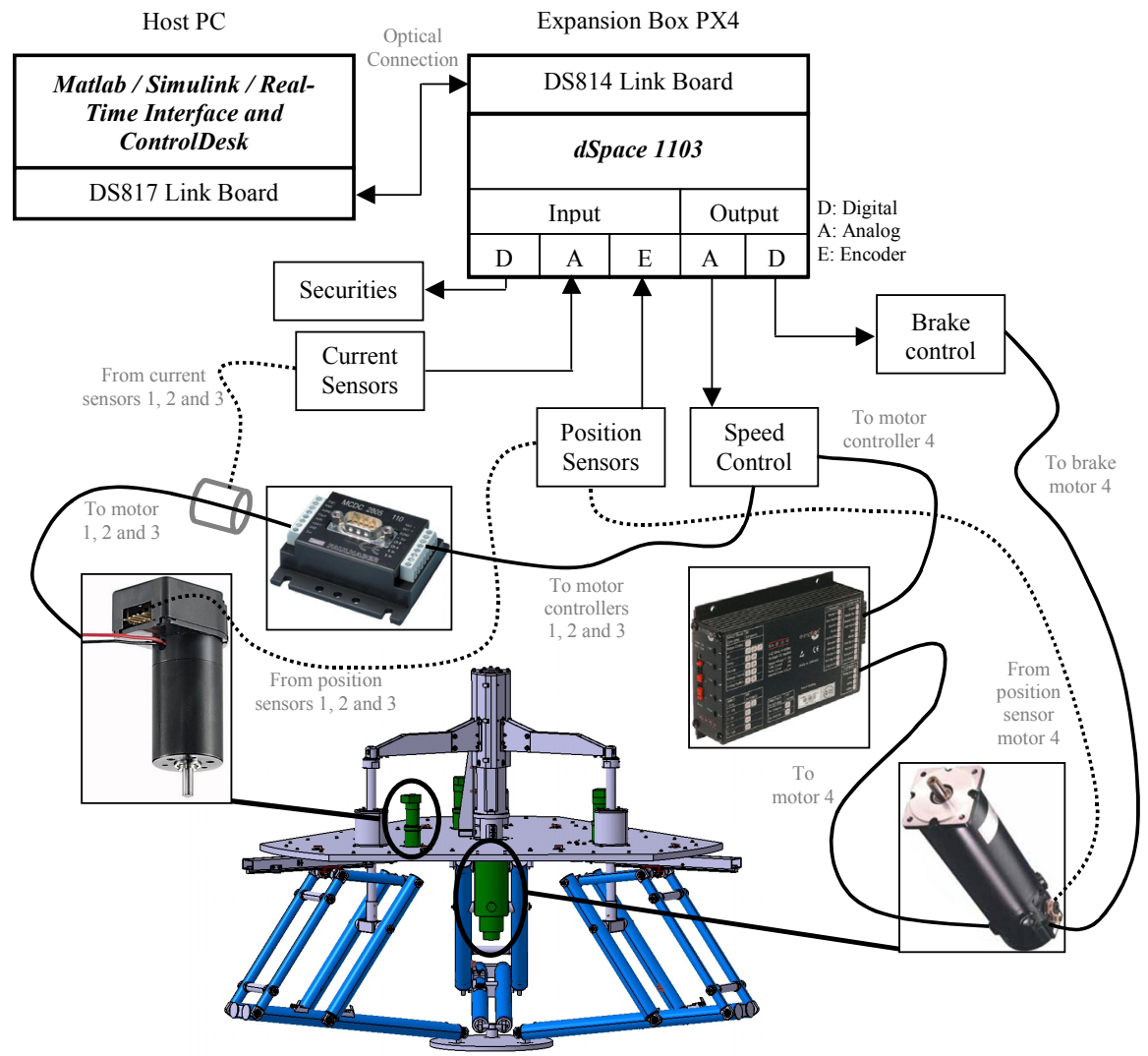

Fig. 10. Experimental bench. 


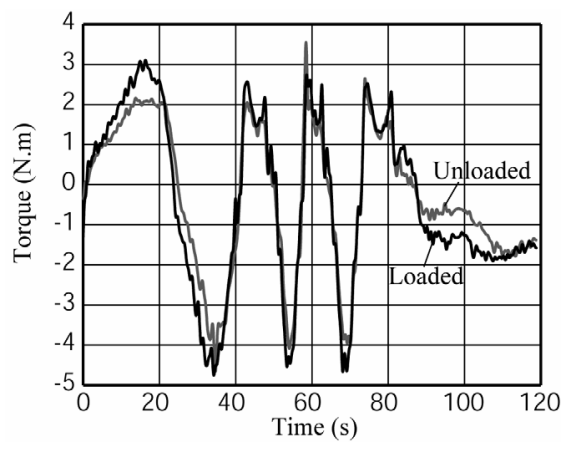

(a) Input torque of actuator $M_{1}$.

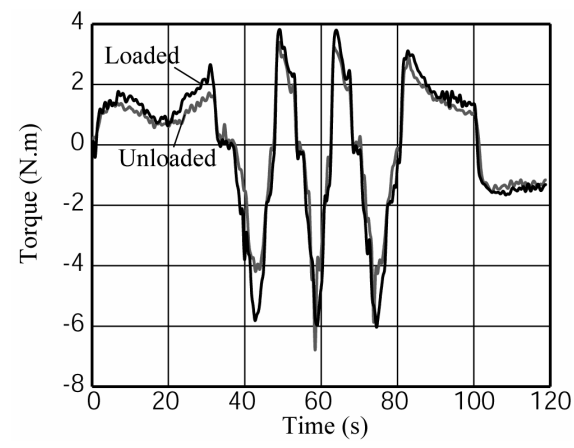

(b) Input torque of actuator $M_{1}$.

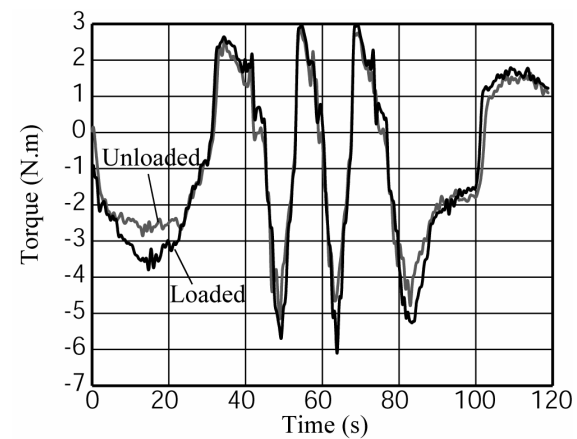

(c) Input torque of actuator $\boldsymbol{M}_{1}$.

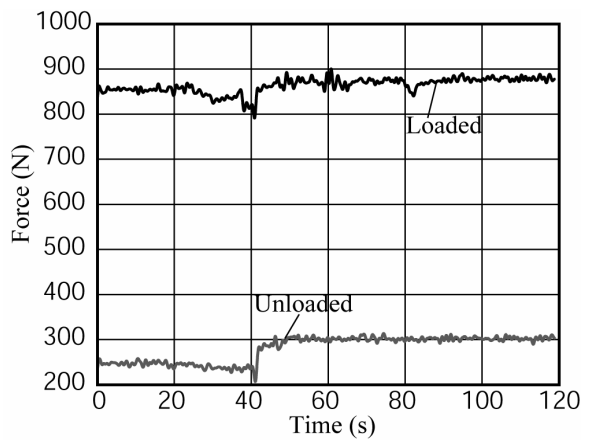

(d) Input torque of actuator $M_{1}$.

Figure 11. Input torques/effort on the actuators with and without payload of $200 \mathrm{~N}$.
Such a design allows the cancellation of the loads of gravity on the actuators, displacing the platform with the payload in the horizontal plane. This property has been validated by experimental tests carried out on the built prototype. It was shown that the input torques of the actuators displacing the platform in the horizontal plane with and without a payload of $20 \mathrm{~kg}$ are similar.

The authors believe that the proposed manipulators may be used in industrial applications for the manipulation of heavy equipment with little power consumption. Use in various fields are possible, depending on the type of industrial application.

Finally, we would like to note that the proposed manipulators have been patented [28] and additional information is available upon request. It should be also noted that on the basis of this design approach similar parallel manipulators with 3 to 6 degrees of freedom and having the same properties may be developed [29, 30].

\section{Acknowledgments}

The authors would like to acknowledge the financial support of Bretagne Valorisation, as well as Professor Jean Le Flecher for the advice during the development of the prototype.

\section{References}

[1] Makino, H., and Furuya, N., 1982, "SCARA robot and its family," In Proceedings of the 3rd International Conference on Assembly Automation, Boeblingen, Germany, pp. 433444.

[2] Clavel., R., 1988, DELTA, a fast robot with parallel geometry. In Proceedings of the $18^{\text {th }}$ International Symposium on Industrial Robot, Lausanne (Switzerland). pp. 91-100.

[3] Clavel., R., 1990, "Device for the movement and positioning of an element in space", Patent US 4976582, December $11^{\text {th }}$, 1990.

[4] Rolland, L., 1999, "The Manta and the Kanuk: Novel 4-DOF Parallel Manipulators for Industrial Handling," In Proceedings of the ASME IMECE'99 Conference, Nashville, USA, November, pp. 831-844.

[5] Pierrot, F., Company, O., Shibucawa, T., Morita., K., 1999, "Four-degree-of-freedom parallel robot." Patent JP26431199, September 17, 1999.

[6] Pierrot, F., Marquet, F., Company, O., and Gil., T., 2001, "H4 Parallel Robot: Modeling, Design and Preliminary Experiments," In Proceedings of the IEEE International Conference on Robotics and Automation, Soul, Korea, pp. 3256-3261.

[7] Angeles, J., Morozov, A., and Navarro, O., 2000, “A Novel Manipulator Architecture for the Production of SCARA motions," in Proceedings of the IEEE International Conference on Robotics and Automation, San Francisco, CA, pp. 2370-2375.

[8] Angeles, J., and Morozov, A., 2005, "Four-degree-offreedom parallel manipulator for producing schonflies motions," Patent US2005262959, December 1, 2005.

[9] Angeles, J., 2005, "The Morphology Design for a Parallel Schönflies-Motion Generator," in Proceedings of the Second International Colloquium on Robotic Systems for Handling and Assembly, Braunschweig, Germany, pp. 37-56. 
[10] Yang, T. L., Jin, Q., Liu, A. X., Yao, F. H., and Luo, Y., 2001, "Structure Synthesis of 4-DOF (3-Translation and 1Rotation) Parallel Robot Mechanisms Based on the Units of Single-Opened-Chain," Proceedings of ASME 2001 Design Engineering Technical Conference and Computers and Information in Engineering Conference, Pittsburgh, PA, Paper No. DETC2001/DAC-21152.

[11] Angeles, J., 2004, "The Qualitative Synthesis of Paralle Manipulators," ASME J. Mech. Des., 126 4), pp. 617-624.

[12] Zhao, T. S., Dai, J. S., and Huang, Z., 2002, "Geometric Synthesis of Spatial Parallel Manipulators With Fewer Than Six Degrees of Freedom,” Proc. Inst. Mech. Eng., Part H: J. Eng. Med., 216(C12), pp. 1175-1186.

[13] Li, Q. C., and Huang, Z., 2003, "Type Synthesis of 4-DOF Parallel Manipulators," in Proceedings of the 2003 IEEE International Conference on Robotics and Automation, Taipei, Taiwan, pp. 755-760.

[14] Carricato, M., and Parenti-Castelli, V., 2004, "On the topological and geometrical synthesis and classification of translational parallel mechanisms." in Proceedings of the 11th World Congress in Mechanism and Machine Science, Tianjin, China, pp. 1624-1628.

[15] Carricato, M., 2005, "Fully Isotropic Four-Degrees-ofFreedom Parallel Mechanisms for Schoenflies Motion,” Int. J. Robot. Res., 24(5), pp. 397-414.

[16] Gosselin, C., Kong, X., Foucault, S., and Bonev, I., 2004, “A Fully Decoupled 3-DOF Translational Parallel Mechanism." In Proceedings of the PKM International Conference, Chemnitz, Germany, pp. 595- 610 .

[17] Kong, X., and Gosselin, C. M., 2004, "Type Synthesis of Input-Output Decoupled Parallel Manipulators," Trans. Can. Soc. Mech. Eng., 28(2A), pp. 185-196.

[18] Gogu, G., 2004, "Fully-Isotropic T3R1-Type Parallel Manipulators," On Advances in Robot Kinematics, J. Lenarčič and C. Galletti, eds., Kluwer Academic, Dordrecht, pp. 265-272.

[19] G. Gogu., G., 2005, "Singularity-Free Fully-Isotropic Parallel Manipulators with Schönflies Motions." In Proceedings of the International Conference on Advanced Robotics, July 18-20, pp. 194-201.

[20] Richard, P.L., Gosselin, C., and Kong, X., 2007, "Kinematic Analysis and Prototyping of a Partially Decoupled 4-DOF
3T1R Parallel Manipualtor," Transaction of the ASME. Journal of Mechanical Design, 129, pp. 611-616.

[21] Arakelian., V., 1998, "Equilibrage des manipulateurs manuels." Mechanism and Machine Theory, 33(4), pp. 437442.

[22] Arakelian, V., 2004, "The history of the creation and development of hand-operated balanced manipulators." In Proceedings of the HMM2004, Kluwer Academic Publishers, pp. 347-356.

[23] Gosselin, C.M. and Angeles, J., (1990), "Singularity analysis of closed-loop kinematic chains." IEEE Transactions on Robotics and Automatics. 6(3), pp.281-290.

[24] Zlatanov, D., Fenton R.G. and Benhabib. B., 1994, "Singularity analysis of mechanisms and robots via a velocity-equation model of the instantaneous kinematics." In Proceedings of the 1994 IEEE International Conference on Robotics and Automation, pp. 980-991.

[25] Bonev, I.A., Zlatanov, D., and Gosselin, C.M., 2003, "Singularity analysis of 3-DOF planar parallel mechanisms via Screw Theory." Transactions of the ASME. Journal of Mechanical Design. Vol. 125, pp. 573-581.

[26] Briot, S., Bonev, I. A., Chablat, D., Wenger Ph., and Arakelian V., "Self Motions of General 3-RPR Planar Parallel Robots", The International Journal of Robotics Research (in press).

[27] Arakelian, V., Guegan, S., and Briot, S., 2005, "Static and Dynamic Analysis of the PAMINSA", Proceedings of the ASME 2005 IDETC/CIE Conference, September 24-28, Long Beach, USA

[28] Arakelian, V., Maurine, P., Briot, S., and Pion, E., 2006, "Parallel robot comprising means for setting in motion a mobile element split in two separate subassemblies", WO 2006/021629, January $27^{\text {th }} 2006$.

[29] Arakelian, V., Briot S., Guegan, S. and Le Flecher J., 2005, "Design and Prototyping of New 4,5 and 6 Degrees of Freedom Parallel Manipulators Based on the Copying Properties of the Pantograph Linkage", Proceedings of the 36th International Symposium on Robotics (ISR), 29 November - 1er December, Tokyo, Japan.

[30] Briot, S., 2007, "Analyze and Optimization of a New Class of Parallel Manipulators with decoupled motions", Ph.D. Dissertation, I.N.S.A. of Rennes, 212p. 